\title{
Research of Diesel Domain Ontology Frame Construction
}

\section{Zhaohua Chen ${ }^{1, \mathrm{a}}$, Jibin $\mathrm{HaO}^{2, \mathrm{~b}}$, Lijian $\mathrm{Hao}^{3, \mathrm{c}}$, Dingguo Sheng ${ }^{4, \mathrm{~d}}$}

\author{
${ }^{1}$ Department of Information and Digital Center, China North Engine Research Institute, \\ Tianjin, 300400, China \\ ${ }^{2}$ Department of Information and Digital Center, China North Engine Research Institute, \\ Tianjin, 300400, China \\ ${ }^{3}$ Department of Information and Digital Center, China North Engine Research Institute, \\ Tianjin, 300400, China \\ ${ }^{4}$ Department of Information and Digital Center, China North Engine Research Institute, \\ Tianjin, 300400, China \\ aemail: 464859729@qq.com, ${ }^{\text {bemail:hassar824@126.com, }{ }^{c} e m a i l: ~}$ \\ haolijian1@163.com, demail:sxdtsdg@163.com
}

\section{Key words: Diesel Domain Ontology, Knowledge Management, Knowledge Retrieval}

\begin{abstract}
The paper aims at knowledge management and application problems during the diesel research process, reviews the development of ontology in the Semantic Web domain, analyzes the requirement of the knowledge ontology frame, puts forward the diesel domain ontology candidate principle, constructs the whole lifecycle of the diesel engine oriented ontology. The ontology construction tool and interface development could be applied to the knowledge searching, which could promote and popularize the knowledge management system.
\end{abstract}

\section{Introduction}

Currently, designers have accumulated large amount of knowledge during the diesel research, but most of these knowledge are raw s scattered and nonstandard, which does not do any systematism、standardization and integration research that aims at diesel domain knowledge, the level of knowledge reuse is relatively low and the professional knowledge accumulation 、 deposition and application is also weak, which has restricted the improvement of design ability. In order to resolve knowledge accurate retrieve and knowledge reuse, the paper has proposed the ontology construction frame that aims at resolving knowledge management and application problem during the diesel design and research process. Through developing ontology construction tools and data parse interfaces, it could achieve knowledge resource application based on the diesel domain ontology.

\section{The development of ontology}

The ontology was first used by Rudolf Goclenius in his Ogdooas Scholastica in 1606. [1] The meaning of the ontology in Oxford English Dictionary is that 'branch of metaphysics concerned with the nature or essence of being or existence.' [2] After that it was used in artificial intelligence and the Semantic Web as the structural frameworks for organizing information.[3] 'The ontology is a formal, explicit specification of a shared conceptualisation',[4] it could be seen as one of the pillars of the Semantic Web.[5] It plays an important role in knowledge management, knowledge sharing, knowledge integration and knowledge analysis. Since the mid-1970s, artificial intelligence researchers realized that to capture knowledge is crucial for building large and powerful AI systems. [6] They believed that new ontologies could be created to implement automated reasoning in computational models. In the 1980's, the AI community tried to utilize the ontology to describe both a theory of modeled world and a component of knowledge systems. [6] After 1984's, computer 
scientists have built general or upper-level ontologies, such as WordNet and Cyc.[7] They tried to assemble a comprehensive ontology, which enable enableartificial intelligence applications to perform human-like reasoning.[6] In 2001, Tim Berners-Lee who was the inventor of the World Wide Web put forward the concept of the Semantic Web. Ontology enabled semantic relations among represented entities and concepts to be expressed based on the Semantic Web.[8]

\section{Demand of the diesel domain ontology construction}

Currently, the normalization of the diesel retieval platform and knowledge searching efficiency is relatively low, the knowledge integration is difficult. Since the ontology is design for shared understanding, facilitate communication, normative models, inter-operability of sharing and reuse.[9] The diesel domain ontology has been introduced to resolve these problems.

The user may have different descriptions for the same diesel terminology, different repositories may have different field names for the same concept, which leads to the difficulty of the repositories integration, such as 'lower crankcase', some users may term for 'oil sump'. For each concrete structure, Chinese standard has explicit terminology and definition, but users may have their habitual term, which conflicts with Chinese standard. Furthermore, different information systems may describe the same concept as different terminologies, it leads to the difficulty of the information integration. For example: information system 1 may term for 'stiffness experimentation', information system2 may term for 'stiffness test'.

The knowledge reasoning is difficult. If users input 'ductile iron', it is difficult for the existing platform to speculate the 'nodular cast iron', as shown in Figure 1.

The diesel ontology frame could resolve the problem that the same diesel terminology has different descriptions. Terminologies in the Chinese standard are used in the diesel ontology frame, some chronic terms have incidence relations stored in the ontology frame.

The existing searching system's knowledge searching efficiency is relatively low. For example, if a user inputs 'crankcase', all of documents relate crankcase will be found, most of knowledge are not what the user wants to get, the user should filter constantly to get the knowledge , which has greatly reduced the efficiency of knowledge acquisition.

In the diesel ontology, each terminology has incidence relations with other terminologies, the user could navigate to the knowledge that they want to get. [10]For example, if a user wants to search the knowledge about the 'connecting rod', in the diesel ontology frame, 'connecting rod' and 'fatigue test' have an incidence relation, if the user inputs 'connecting rod', it could relate to the 'fatigue test'. Building the incidence relation of diesel terminology could improve the accuracy of the knowledge retrieve, and thereby improve the efficiency of the knowledge retrieve.[11]

Input The known material is the ductile iron

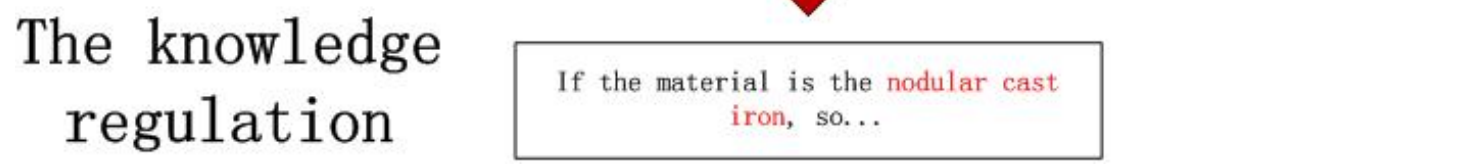

Fig.1. Knowledge deduce is difficult

\section{Methodology of diesel domain ontology}

According to the diesel application requirement analysis of diesel ontology, the aim of the ontology construction is to resolve the same concept that has different descriptions, it could also improve the accuracy of knowledge searching. Furthermore, researching different knowledge points 
could also improve knowledge searching efficiency. Thus our research content mainly concludes the methodology of the diesel domain ontology, ontology construction tools and whole lifecycle oriented domain ontology.

\subsection{The construction of the diesel ontology model frame}

The diesel ontology model could be divided into knowledge standard ontology v knowledge synonymy ontology and knowledge candidate ontology, figure 2 has shown the relation.

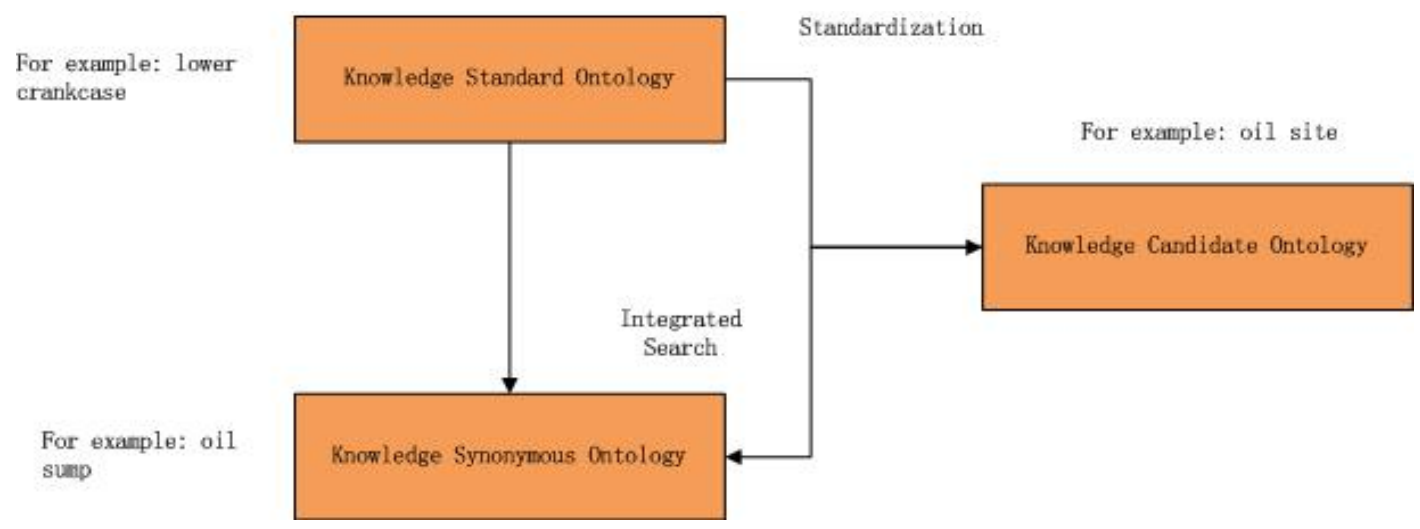

Fig.2. Diesel ontology model

The diesel ontology model mainly includes class、 relation、 function、axiom and instance these five elements.[12]

\section{Class}

The meaning of class is wide, it could point to aught, such as work description 、 behavior 、 strategy and deduction process. From the semantic perspective, the concept represents the aggregate of objects, its definition adopts frame structure. The class of the diesel ontology has structure class 、 exception class、 experimentation class and so on.[13]

\section{Relation}

The relation represents the interaction between concepts in a domain, formally defines n-dimensions subclass of the Cartesian product: $\mathrm{R}: C_{1} \times C_{2} \times \ldots \times C_{\mathrm{n}}$.From the semantic perspective, relation corresponds to the aggregate of object element.[14] There are four basic relations between different concepts, as shown in the Table1. In the actual modeling process, relations between different concepts are not limit to these four basic relations, which could depend on the specific situation of domain to define corresponding relations. [15]

\begin{tabular}{|l|l|}
\hline Relation Name & Relation Description \\
\hline Part-of & $\begin{array}{l}\text { Represent different consepts'relations between part and whole } \\
\text { Khich is similar as the object oriented relations between supper } \\
\text { class and subclass }\end{array}$ \\
\hline Instance-of & $\begin{array}{l}\text { Represent relations between concept and concept's relation, } \\
\text { which is similar as the object oriented relations between class } \\
\text { and instance }\end{array}$ \\
\hline Attribute-of & $\begin{array}{l}\text { Represent a certain concept is an attribute of another concept. } \\
\text { For example, 'Concept' is an attribute of 'structure' }\end{array}$ \\
\hline
\end{tabular}

\section{Function}

Tab.1.Relation of the ontology model

Function is a special relationship. In this relationship, the first $n-1$ elements can uniquely determine the Nth element, which formally defined as $\mathrm{F}: c_{1} \times C_{2} \times \ldots \ldots \times C_{n-1} \rightarrow c_{n}$ if father-of is a function, father-of $(x, y)$ means $y$ is the father of X.[16]

\section{Axiom}

The Axiom represents a permanent true assertion.[16] 


\section{Instance}

The instance represents the element. From the semantic point of view, the instance represents the objects, [17] it is a certain class that reflected in the real world.

The construction of diesel ontology, design 、material arts and crafts vexperimentation 、 test faulty and maintain should be considered. The figure 3 has shown the hierarchy of each dimension.

\subsection{The diesel ontology construction principle}

The construction of the diesel ontology could help users rapidly comprehend the knowledge about diesel and improve accuracy of the knowledge retrieve. Thus, the construction of the diesel ontology should follow principles as follows:

Principle1 Reducing the ontology terminology and combination as much as possible, search the knowledge should be as accurate and comprehensive as possible.

Principle2 The structure of programming should spread out layer by layer according to the nature of the ontology, which makes it easy to the inherence of the classes' parameters.

Principle3 Ontology minimum covering principle. In the knowledge instance, not each terminology could be utilized in the ontology, only the terminology occurs a certain amount in different knowledge instances, it could be considered as the knowledge ontology.

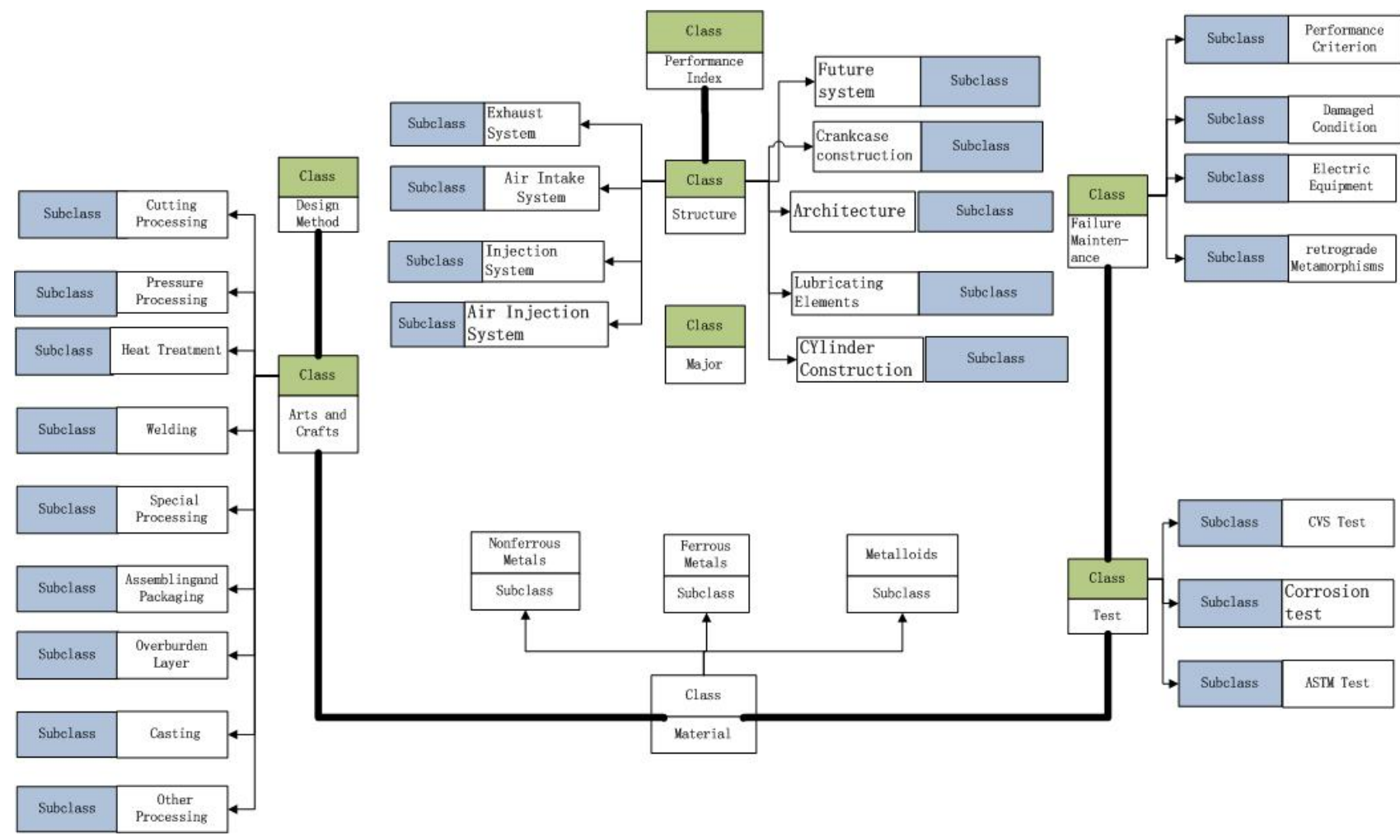

Fig.3.Diesel ontology frame

Principle4 Requirement-driven principle. The construction of the ontology is the requirement-driven, the requirement is the knowledge instance, which could build different dimensions ontology through the requirement of the knowledge instance searching.

Principle5 Support the relevant knowledge's overall searching.

\subsection{Knowledge ontology case}

The Crankcase ontology is as shown in Figure 4.

The terminology in the full line frame is the ontology utilized to repository for retrieving, the terminology in the dashed line frame is the ontology utilized to relevancy retrieve. 


\section{The application of the diesel domain ontology}

We have developed a suit of knowledge ontology management tools for the sake of retrieve application based on the diesel domain ontology, which includes: knowledge ontology repository management 、 knowledge ontology terminology edit management 、 knowledge ontology terminology map 、 ontology terminology application management 、 knowledge ontology terminology interface and other functions. These functions are based on international standard OWL ontology description、 parsing technique and ontology editor Protégé, it adopts standard SSH

(Spring、Struts、Hibernate) framework technology to achieve uploading ontology relevancy 、 diesel ontology visual display v knowledge retrival based on the ontology.

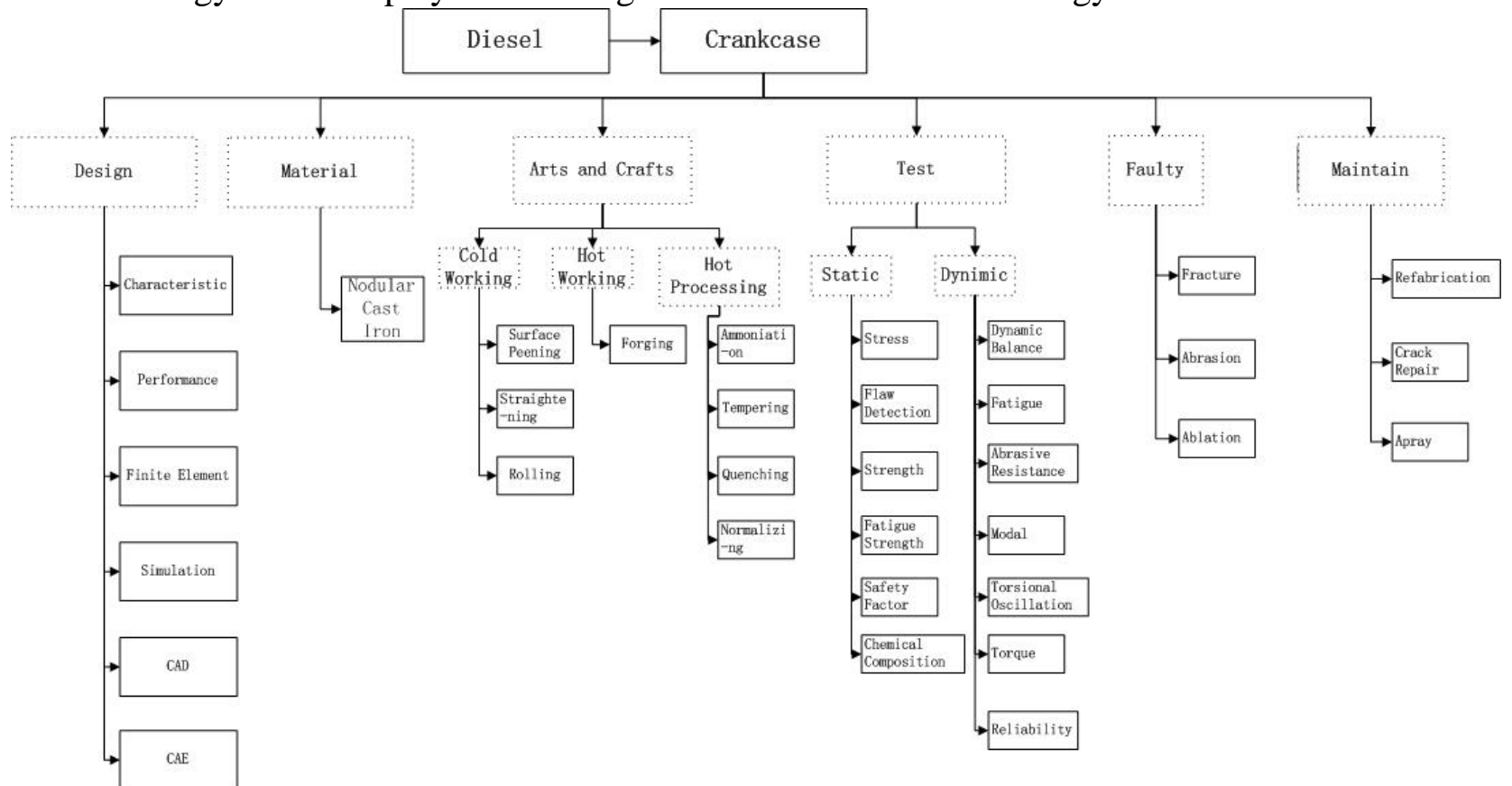

Fig.4.Crankcase ontology

\subsection{The knowledge retrieval based on the synonymy ontology}

The synonymy ontology fuses the same concept which has different description names. For example: The knowledge about "dynamic balance" and "inertia force balance” are the same kind of knowledge, which could do relevant retrieve. The figure 5 has shown the knowledge retrieve based on the synonymy ontology 


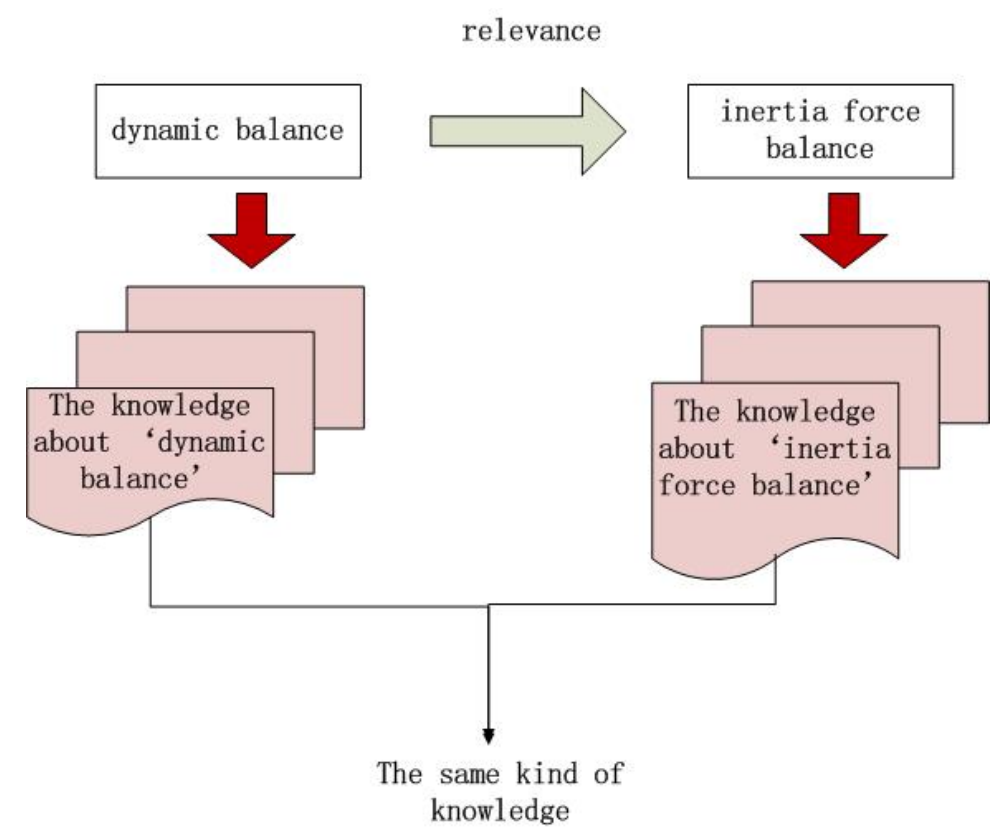

Fig.5.Knowledge retrieve based on the synonymy ontology

\subsection{The relevant knowledge retrieval base on the ontology}

To utilize the relations between knowledge ontology, to fuse the relevant knowledge and provide users with classification could depress users' retrieve workload remarkablely. For example, if an user input 'crankshaft', the system could deliver relative knowledge such as design 、 material、arts and crafts vexperimentation、 test、faulty 、maintain and so on, from which, users could choose some useful knowledge.

\subsection{The knowledge exhibition base on the ontology meshwork}

The ontology meshwork not only shows the complex relations between different knowledge ontologies, such as similarity relation v progressive relation, the complex relations between different knowledge could also be shown through knowledge ontology meshwork.

\section{Conclusion}

The essay does a deeply research based on the ontology construction technology, which combined with the demand of existing platform, puts forward diesel ontology structure model frame、 diesel knowledge candidate ontology primary election principle, constructs the whole lifecycle oriented diesel domain ontology. Through developing ontology construction tool and interfaces in knowledge retrieval application, it could comprehensively promote the spreading and application of knowledge management system. 


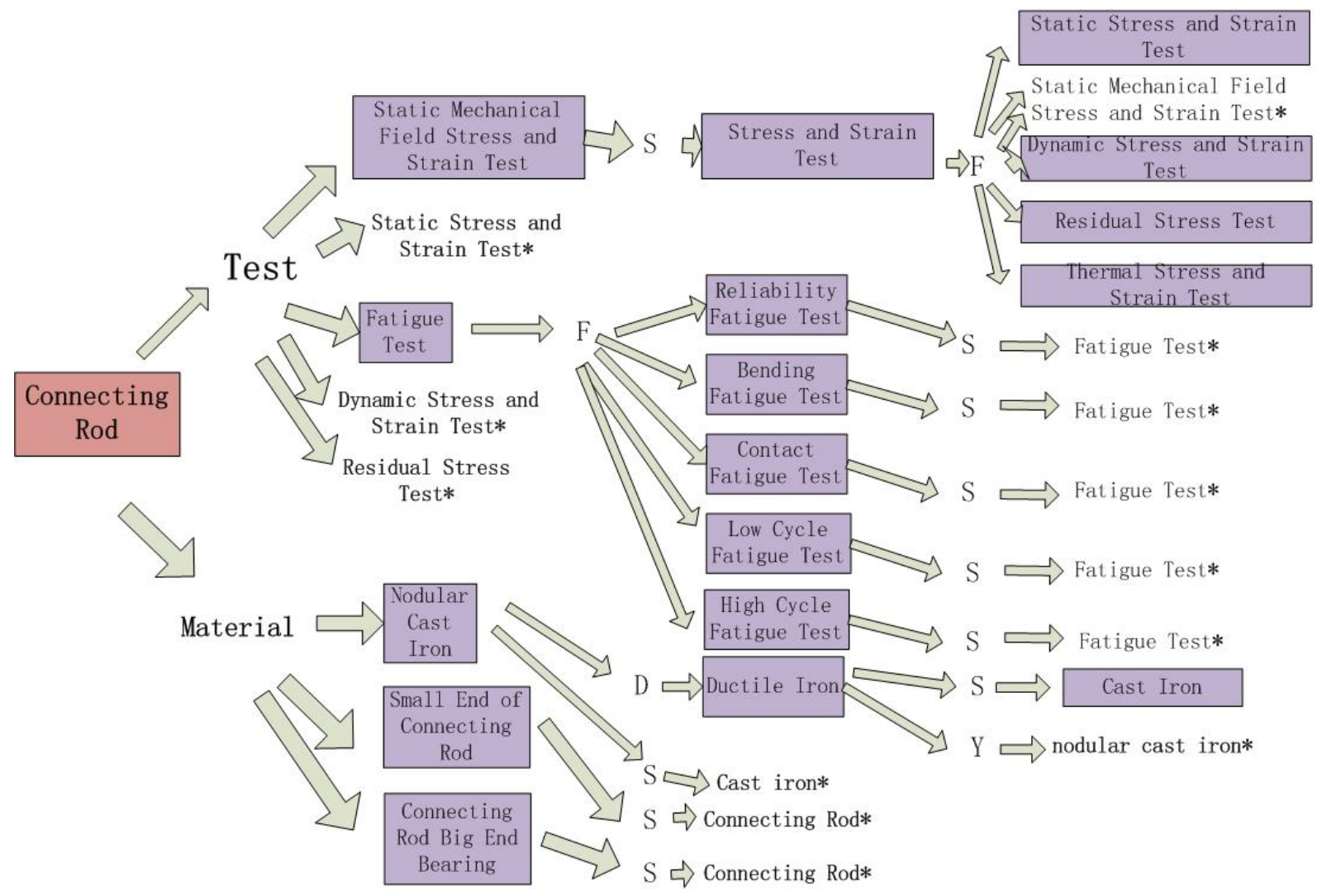

Fig.6.Knowledge exhibition based on the ontology

\section{Reference}

[1] Ontology: Its Role in Modern Philosophy, 2011. Theory and History of Ontology. [online] Available at: <http://www.ontology.co/> [Accessed 5 May 2015 ].

[2] Simpson. J.,Weiner. E., 2004. The Oxford English Dictionary. Oxford: Oxford University Press.

[3] Open Structs Tech Wiki, 2011. Intro to Ontologies. [online] Available at: <http://techwiki.openstructs.org/index.php/Intro_to_Ontologies> [Accessed 1 May 2015 ].

[4] Gruber.T.R., 1993. A Translation Approach to Portable Ontology Specifications, 5(2), p.199-220.

[5] Semantic Web, 2012. Ontology. [online] Available at: <http://semanticweb.org/wiki/Ontology> [Accessed 5 May 2015].

[6] Gruber, T. (2008). "Ontology". In Liu, Ling; Özsu, M. Tamer. Encyclopedia of Database Systems (Springer-Verlag). ISBN 978-0-387-49616-0.

[7] Salay. N., Witbrock. M., 2002. Inducing criteriafor massnounlexical mappings using the CycKB, and its extension to WordNet, [e-journal] 42(6), p 23.

[8] Zhang, J. (2007). Ontology and the Semantic Web. Proceedings of the North American Symposium on Knowledge Organization. Vol. 1. Available at<: http://dlist.sir.arizona.edu/1897/>

[9] W3C, 2013. W3C Semantic Web Technology. [online] Available at: <http://www.w3.org/2001/sw/> [Accessed 1 May 2015].

[10] Hedden Information Management, 2013. Taxonomies, Thesauri, and Controlled Vocabularies[pdf] [online] Available at: <http://www.hedden-information.com/taxonomies.htm> [Accessed 1 May 2015].

[11] Chandrasekaran. B. , Josephson.R , 2008, What Are Ontologies, and Why Do We Need 
Them.[online]

Available

at:

<http://www.csee.umbc.edu/courses/771/papers/chandrasekaranetal99.pdf> [Accessed 1 May 2015].

[12] Pascal Hitzler, Markus Kr"otzsch, Bijan Parsia, Peter F. PatelSchneider, and Sebastian Rudolph, editors. OWL 2 Web Ontology Language: Primer.

W3C Recommendation, 2009. Available at< http://www.w3.org/TR/owl2-primer/.>

[13] Pascal Hitzler, Markus Kr"otzsch, and Sebastian Rudolph. Foundations of Semantic Web Technologies. Chapman \& Hall/CRC, 2009.

Baader, F., Lutz, C., Milicic, M., Sattler, U., and Wolter,

[14] F. Integrating Description Logics and Action Formalisms: First Results. In Proc. of the 20th National Conference on Artificial Intelligence(AAAI-05) (2005), A. Press, Ed.

[15] [Baader et al., 2007] Franz Baader, Diego Calvanese, Deborah McGuinness, Daniele Nardi, and Peter Patel-Schneider, editors. The Description Logic Handbook: Theory, Implementation, and Applications. Cambridge University Press, second edition, 2007.

[16] I. Horrocks and U. Sattler. Ontology reasoning in the SHOQ(D) description logic. In Proc. ofIJCAI 2001, pages 199-204, 2001

[17] K. Schild. A correspondence theory for terminological logics: Preliminary report. In Proc. ofIJCAI'91, pages 466-471, 1991. 